\title{
RESEARCH OF THE WRINKLING ELIMINATION OF STAINLESS STEEL SUS304 BY VISCOUS PRESSURE
} Tiejun Gao ${ }^{1 *}$-Kun $\mathrm{Yu}^{2}$-Xiaojun Liu ${ }^{1}$-Hepeng Zhang ${ }^{1}$

\author{
${ }^{1}$ Faculty of Aerospace Engineering, Shenyang Aerospace University, No. 37 Daoyi South Avenue, Shenyang 110136 \\ Liaoning, China \\ ${ }^{2}$ AECC Shenyang Liming Aero engine co., LTD, No.6 East Street, Shenyang 110043 Liaoning, China
}

\begin{tabular}{l} 
ARTICLE INFO \\
\hline Article history: \\
Received: 16.8 .2016$. \\
Received in revised form: 6.3 .2017$. \\
Accepted: 27.3 .2017$. \\
\hline Keywords: \\
Wrinkling \\
YBT \\
Viscous pressure forming \\
Wrinkling elimination \\
\hline DOI:
\end{tabular}

DOI: http://doi.org/10.30765/er.39.2.3

\begin{abstract}
:
Wrinkling is one of the most important factors influencing a forming precision of sheet metal, which brings difficulties to the forming process of sheet metal. In order to eliminate the wrinkling during the forming process, an accurate prediction is necessary. In this paper, the wrinkling elimination process was investigated based on the principle of the Yoshida Buckling Test (YBT) and viscous pressure forming. The experimental device was designed, and evaluation method of the wrinkling elimination rate was presented by the stainless steel SUS304. On this basis, the wrinkling elimination experiment was carried out, the influences of both the viscous medium molecular weight and the tensile state of wrinkle under the viscous pressure on the wrinkling elimination were obtained.
\end{abstract}

criterion, strain rate catastrophe criterion, and appropriate forming parameters or forming methods. Meanwhile, wrinkling is caused by the compression instability, which is influenced by many factors such as forming craft, material property, and stress state. The wrinkling of the sheet metal with complicated shape is difficult to predict accurately during the forming process. Methods for avoiding wrinkling are various depending on different forming processes. A blank holder is employed to prevent wrinkling in the flange area during the drawing process, while for the wrinkling during the tube bending, an adding filler inside the tube can effectively prevent wrinkling [5-8]. If wrinkling is allowed at certain conditions during the forming process, as long as the change of a wrinkle can be controlled to fit the mold before the end of deformation, the size precision can meet the requirements. In recent years, tube-compression by viscous pressure forming aims at controlling the

\footnotetext{
${ }^{*}$ Corresponding author. Tel.: +8613504999851;

E-mail address:tiejun_gao@163.com.
} 
variety of wrinkling states and eliminating the wrinkles of tube-blank, forming parts with large deformation accordingly. Therefore, the study on the wrinkling elimination process and the ultimate elimination ability are of great significance for the optimization of the forming process parameters and the improvement of the ultimate deformation ability of sheet metal [9-14].

The YBT (Yoshida Buckling Test) is a method which can use the diagonal tension to evaluate the ability of resisting wrinkling under non-uniform tension This has an important representative of resisting wrinkling in the forming process of the sheet metal. In this paper, based on the principle of the YBT and viscous pressure forming, the wrinkling elimination process and the degree of ultimate elimination were analyzed under different tensile forces and viscous properties. This study can provide a reference for the ability of the wrinkling elimination of sheet metal characterized with complicated shape by viscous pressure forming [1518].

\section{The experimental Scheme and Materials}

\subsection{The experimental scheme}

During the forming process of sheet metal, stress state and location of wrinkles are difficult to control, which brings difficulties to get the wrinkles of same morphology. In order to simplify the research, the morphology similar to the wrinkle is formed through the mold with a die cavity length of $60 \mathrm{~mm}$ and width of $20 \mathrm{~mm}$ as shown in Fig.1, and the shape of wrinkle can be controlled by the forming pressure.

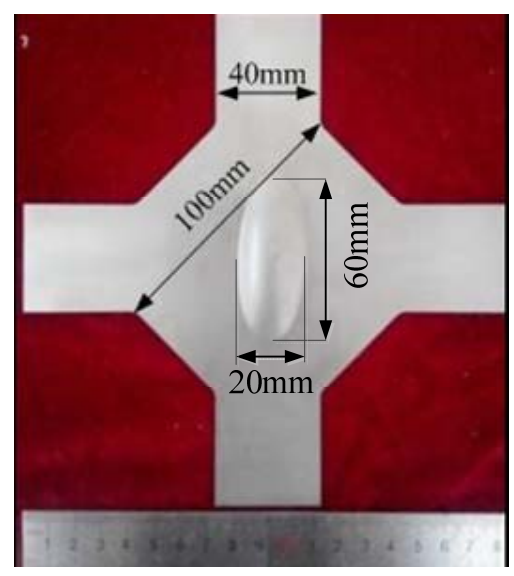

Figure 1. Wrinkling specimen.

\subsection{The experimental device}

The experimental device was designed (Fig.2), which can eliminate wrinkles by viscous pressure. The biaxial tension was loaded along the $\mathrm{X}$ and $\mathrm{Y}$ directions on the wrinkling specimen by the drive ball screw controlled by a servo motor. The viscous pressure was loaded along the $\mathrm{Z}$ direction by a servo motor.

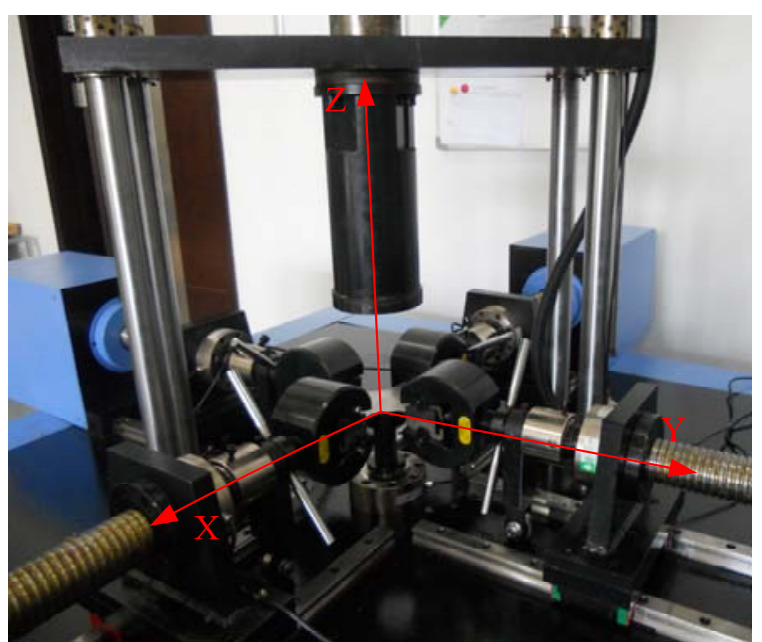

Figure 2. The experimental device.

\subsection{The experimental materials}

The sheet metal of stainless steel SUS304 with the thickness of $0.7 \mathrm{~mm}$ was used in the experiment. The tensile test was carried out according to GB/T228.12010. In the experiment, the tensile speed is $10 \mathrm{~mm} / \mathrm{min}$. The experiment was repeated three times as shown in Fig.3. The tensile test data are shown in Table 1. It can be seen that the average load of the specimen, the tensile strength, the yield strength and the elongation are $9.4 \mathrm{kN}, 588.3 \mathrm{MPa}$, $235.1 \mathrm{MPa}$ and at $67 \%$, respectively.

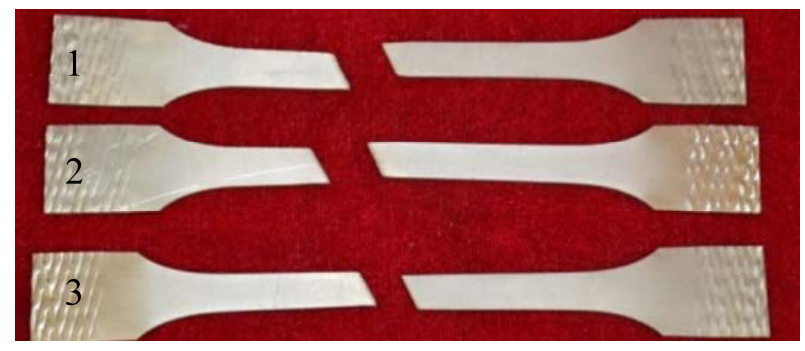

Figure 3. Tensile specimens. 
Table 1. Stainless steel SUS304 tensile test data

\begin{tabular}{ccccc}
\hline Sample & $\begin{array}{c}\text { Maximum load } \\
(\mathrm{kN})\end{array}$ & $\begin{array}{c}\text { Tensile strength } \\
(\mathrm{MPa})\end{array}$ & $\begin{array}{c}\text { Yield strength } \\
(\mathrm{MPa})\end{array}$ & $\begin{array}{c}\text { Fracture elongation } \\
(\%)\end{array}$ \\
\hline Sample 1 & 9.5 & 597.3 & 239.3 & 66.7 \\
Sample 2 & 9.3 & 582.3 & 230.7 & 68.4 \\
Sample 3 & 9.3 & 585.5 & 235.3 & 65.9 \\
Average & 9.4 & 588.3 & 235.1 & 67.0 \\
\hline
\end{tabular}

Methyl vinyl silicone rubber is used as viscous medium in the experiment. It is the viscous plastic linear polymer which is copolymerized with dimethyl siloxane and little vinyl silicone. The molecular structural formula of the methyl vinyl silicone rubber is illustrated in Fig.4. The main chain is composed of silicon and oxygen atoms The pendant groups connected with silicon are methyl and vinyl. The molecular weights of viscous medium are 40, 50, 60 and $70 \times 10^{4} \mathrm{~g} / \mathrm{mol}$ respectively. The relationships between the shear strain rate and the shear stress of the viscous medium are shown in Fig.5. Moreover, when the molecular weight of the viscous medium is higher, the viscous medium will become more sensitive. The shear viscosity with different molecular weights is shown in Fig.6. The lowest shear viscosity is 12.8 $\mathrm{kPa} \cdot \mathrm{s}$ when the molecular weights of viscous medium is $40 \times 10^{4} \mathrm{~g} / \mathrm{mol}$, and the shear viscosity is increased to $25.3 \mathrm{kPa} \cdot \mathrm{s}$ when the molecular weights is $70 \times 10^{4} \mathrm{~g} / \mathrm{mol}$. It can be seen that the shear viscosity increases with the increasing of molecular weight of the viscous medium.

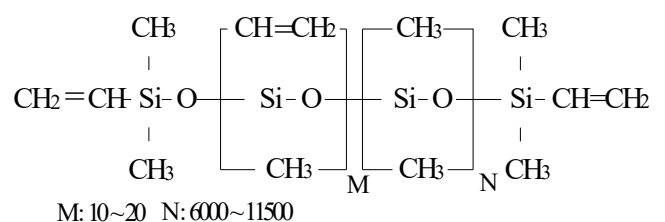

Figure 4. Molecular structure of viscous medium.

\section{Analysis of wrinkling elimination}

\subsection{The stress strain state analysis}

The stress and strain state of the wrinkling under viscous pressure is shown in Fig.7. Besides the viscous pressure, wrinkling was also influenced by a tensile or compression stress along length and width directions of the specimen during the wrinkling elimination process. Considering the force transmission process, only the tensile forces along length and width directions were analyzed. (The tensile force along the length direction is represented by $F_{\mathrm{L}}$ and the tensile force along the width direction is represented by $F_{\mathrm{W}}$ ).

\subsection{The wrinkling elimination method}

In the process of wrinkling, the evaluation method of wrinkle depends on the maximum height of

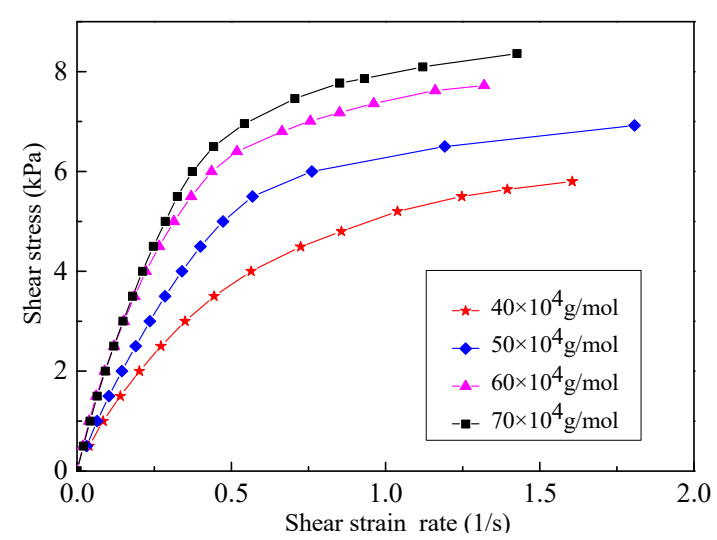

Figure 5. Relationship between shear strain rate and shear stress of the viscous medium.

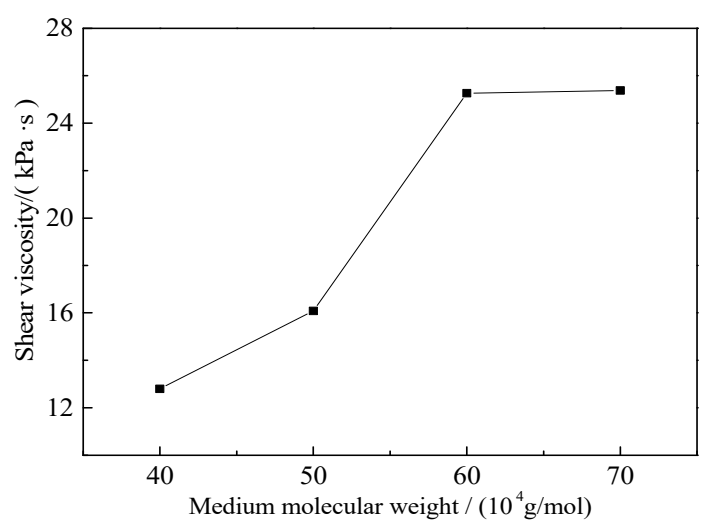

Figure 6. Shear viscosity with different medium molecular weights. 
wrinkling $H_{\max }$, the maximum tilt angle $\theta_{\max }$, the change of tilt angle $\Delta \theta$ and the minimum curvature radius $\rho_{\min }$ as shown in Fig. 8 [19-20]. In the process of wrinkling elimination, some wrinkles can be eliminated completely, but others still have residual height. Considering the macroscopic characteristics and the surface quality factor after the wrinkling elimination, height change of wrinkle is used as the evaluation criteria of wrinkling elimination rate in the process of research.

$$
\beta=H / H_{\max }
$$

where $\beta$ is wrinkling elimination rate, $H$ is residual height after wrinkling elimination $(\mathrm{mm})$ and $H_{\max }$ the maximum height of wrinkle $(\mathrm{mm})$.

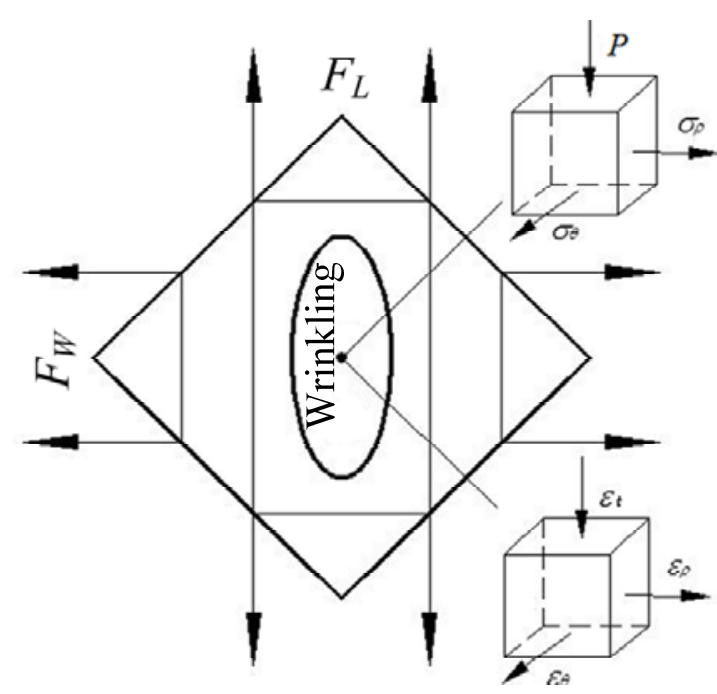

Figure 7. Stress and strain state of the wrinkling by the viscous pressure.

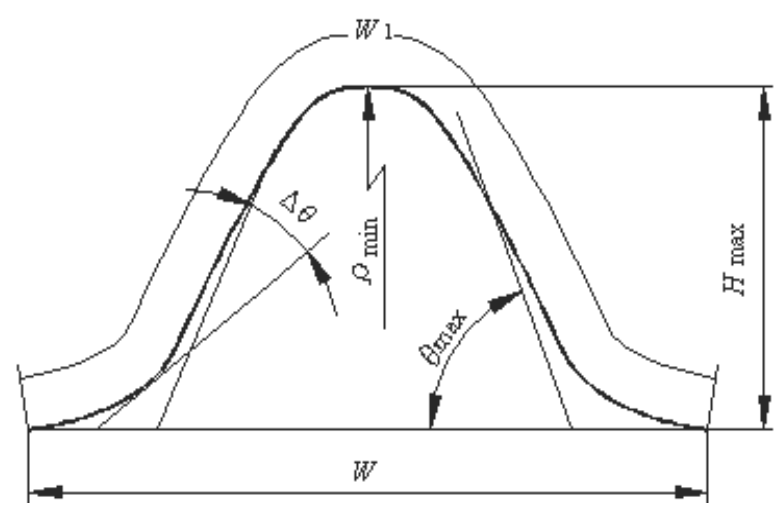

Figure 8. The evaluation method of wrinkle morphology.

\section{The results and discussions}

\subsection{Influence of the molecular weight of viscous medium on the wrinkling elimination}

The results of the wrinkling elimination without tensile force under different molecular weight conditions were measured by height gauge, as shown in Fig. 9. The height of the wrinkle is $4 \mathrm{~mm}$, and the viscous pressure for wrinkling elimination is $30 \mathrm{MPa}$. The relationship between the molecular weight and the wrinkling elimination rate is shown in Fig.10. The maximum displacement of wrinkling peak is $3.43 \mathrm{~mm}$, and the wrinkling elimination rate is $85.8 \%$ at the molecular weight of $40 \times 10^{4} \mathrm{~g} / \mathrm{mol}$. The maximum displacement of peak of the wrinkle is $3.56 \mathrm{~mm}$, and the wrinkling elimination rate is $89.2 \%$ at the molecular weight of $70 \times 10^{4} \mathrm{~g} / \mathrm{mol}$.

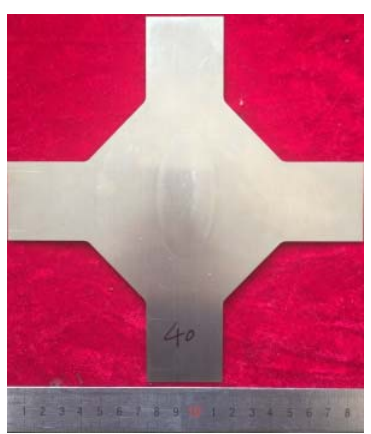

(a) $40 \times 10^{4} \mathrm{~g} / \mathrm{mol}$

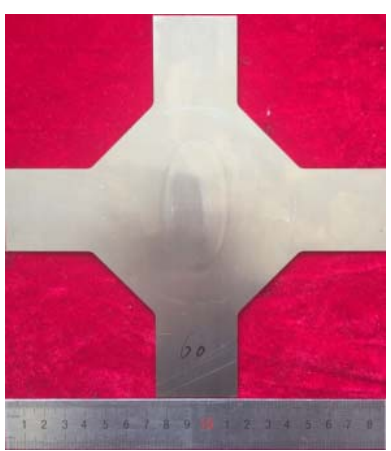

(c) $60 \times 10^{4} \mathrm{~g} / \mathrm{mol}$

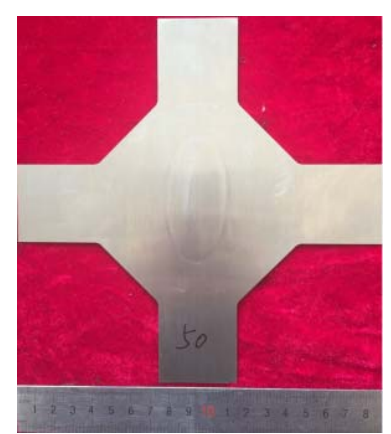

(b) $50 \times 10^{4} \mathrm{~g} / \mathrm{mol}$

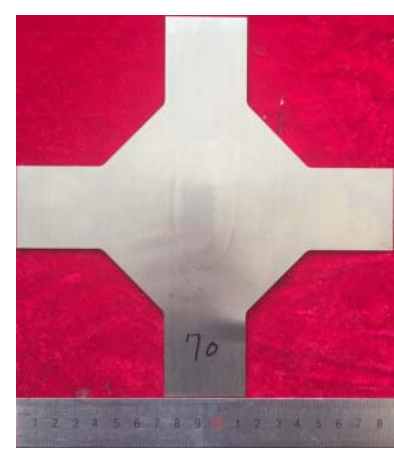

(d) $70 \times 10^{4} \mathrm{~g} / \mathrm{mol}$
Figure 9. Wrinkling elimination specimens with different molecular weights of viscous medium.

Relatively, the wrinkling elimination rate is increased by $3.4 \%$. It can be seen that the wrinkling elimination rate will be increased when the viscous 
medium with larger molecular weight is used. Therefore, the viscous medium with molecular weight of $70 \times 10^{4} \mathrm{~g} / \mathrm{mol}$ will be used in the following experiment.

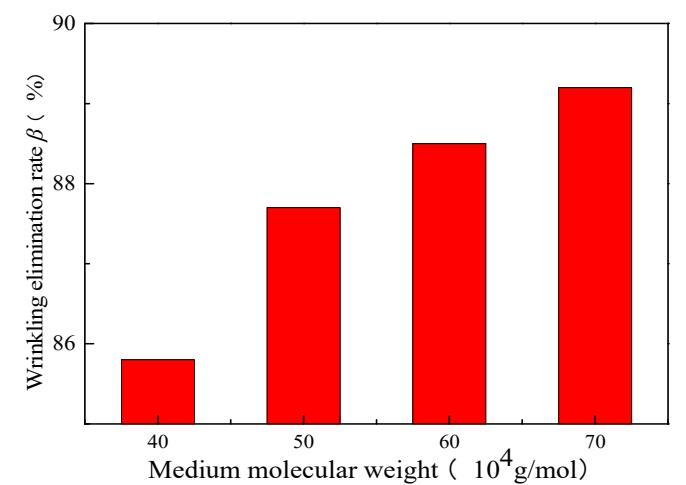

Figure 10. Relationship between the molecular weight of viscous medium and the wrinkling elimination rate.

\subsection{Influence of tensile state on the wrinkling elimination}

Considering the influence of tensile state on the wrinkling elimination, under the experimental condition, different forces were applied directly along the length and width directions, and viscous pressure was loaded to $30 \mathrm{MPa}$. The process parameters of the wrinkling elimination under the biaxial tension condition are shown in Table 2.

The specimens of the wrinkling elimination are shown in Fig.11. The wrinkling elimination rate is $96 \%$ when $F_{\mathrm{W}}=140 \mathrm{~N}$ and $F_{\mathrm{L}}=56 \mathrm{~N}$. When $F_{\mathrm{W}}=140$ $\mathrm{N}$ and $F_{\mathrm{L}}=112 \mathrm{~N}$, the wrinkling elimination rate is $96.6 \%$, which is increased by $0.6 \%$. The wrinkling elimination rate is $94.6 \%$ when $F_{\mathrm{L}}=140 \mathrm{~N}$ and $F_{\mathrm{W}}=56 \mathrm{~N}$. When $F_{\mathrm{L}}=140 \mathrm{~N}$ and $F_{\mathrm{W}}=112 \mathrm{~N}$ the wrinkling elimination rate is $95.7 \%$, which is increased by $1.1 \%$. The relationships between the wrinkling elimination rate and the variations of tensile forces are shown in Fig.12. It can be seen that the tensile force along the width direction has a more significant influence on the process of the wrinkling elimination. It is because the tensile force is easier to transmit and wrinkling is easier to extend along the width direction, which is helpful in the wrinkling elimination.

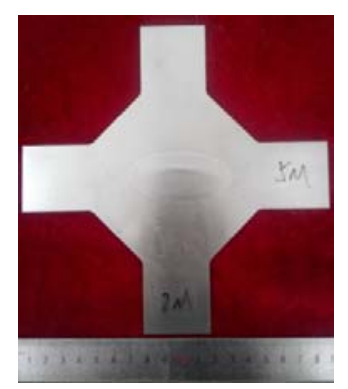

(a) $F_{\mathrm{L}}=140 \mathrm{~N}, F_{\mathrm{W}}=56 \mathrm{~N}$

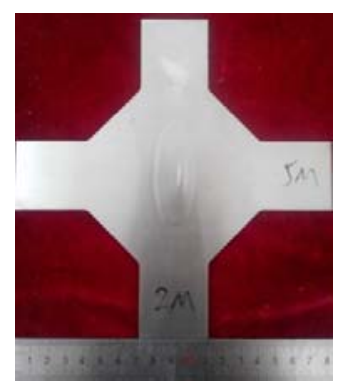

(d) $F_{\mathrm{L}}=56 \mathrm{~N}, F_{\mathrm{W}}=140 \mathrm{~N}$

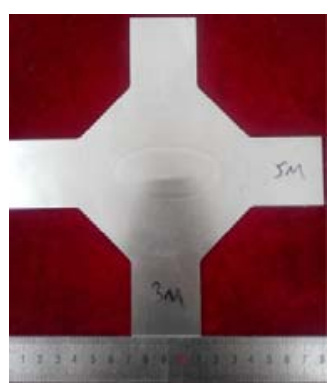

(b) $F_{\mathrm{L}}=140 \mathrm{~N}, F_{\mathrm{W}}=112 \mathrm{~N}$

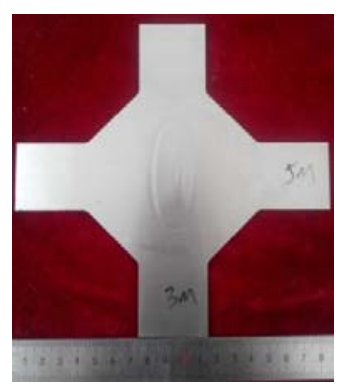

(e) $F_{\mathrm{L}}=84 \mathrm{~N}, F_{\mathrm{W}}=140 \mathrm{~N}$

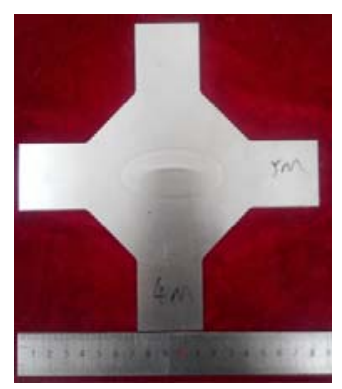

(c) $F_{\mathrm{L}}=140 \mathrm{~N}, F_{\mathrm{W}}=112 \mathrm{~N}$

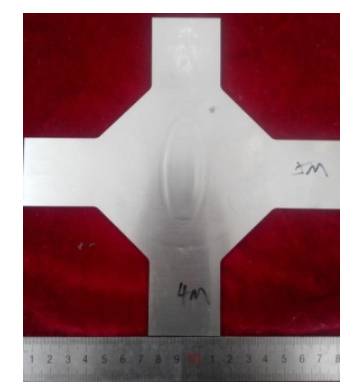

(f) $F_{\mathrm{L}}=112 \mathrm{~N}, F_{\mathrm{W}}=140 \mathrm{~N}$

Figure 11. The wrinkling elimination specimens with different tensile forces along the length and width directions. 
Table 2. The process parameters of the wrinkling elimination under biaxial tension

\begin{tabular}{cccc}
\hline $\begin{array}{c}\text { Viscous } \\
\text { pressure }\end{array}$ & $\begin{array}{c}\text { Tensile force along the length } \\
\text { direction of wrinkle } \\
F_{\mathrm{L}} /(\mathrm{N})\end{array}$ & $\begin{array}{c}\text { Tensile force along the width } \\
\text { direction of wrinkle } \\
F_{\mathrm{W}} /(\mathrm{N})\end{array}$ & $\begin{array}{c}\text { The wrinkling } \\
\text { elimination rate } \\
\beta /(\%)\end{array}$ \\
\hline 30 & 140 & 56 & 94.6 \\
30 & 140 & 84 & 95.3 \\
30 & 140 & 112 & 95.7 \\
30 & 56 & 140 & 96.0 \\
30 & 84 & 140 & 96.3 \\
30 & 112 & 140 & 96.6 \\
\hline
\end{tabular}

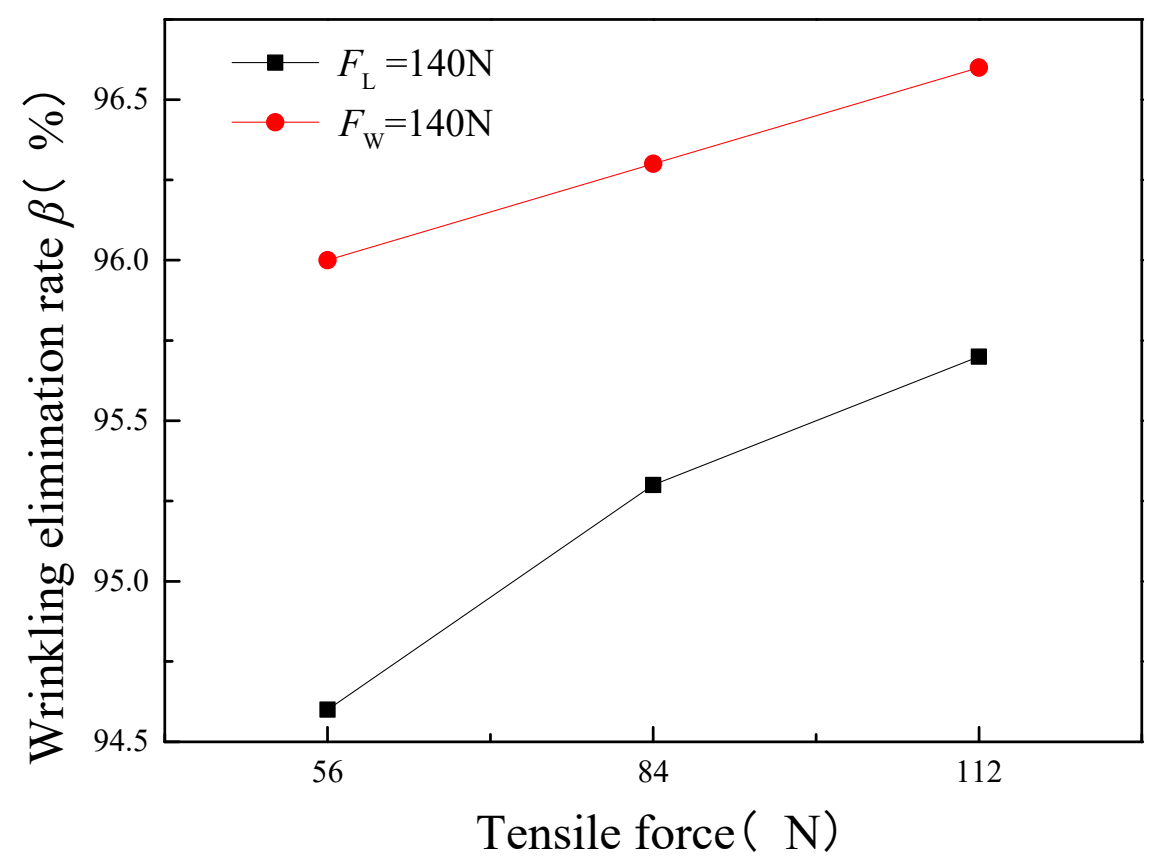

Figure 12. The relationships between the wrinkling elimination rate and tensile state of wrinkle.

\section{Conclusion}

Aiming at the problem of wrinkling of sheet metal, a certain geometrical wrinkle was formed according to the principle of the YBT, and the wrinkling elimination under the condition of viscous pressure was investigated. The results can be extracted as follows:

(1) At the same condition of viscous pressure, when the molecular weight is larger, the viscous medium becomes more sensitive, and higher wrinkling elimination rate can be obtained.

(2) At the same condition of viscous pressure, the tensile state of wrinkle plays a great influence on the wrinkling elimination rate. When the tensile force becomes greater, the wrinkling elimination rate is higher.

(3) Compared with the length direction of the wrinkle, the tensile force exerted at the width direction is beneficial to the transmission of tension, which has a more significant influence on the wrinkling elimination.

\section{Acknowledgments}

The financial support provided by the National Natural Science Foundation of China (51205260) and supported by Program for Liaoning Excellent Talents in University (LJQ2015083) are gratefully acknowledged. 


\section{References}

[1] Hsu, C.W., Ulsoy, A.G., Demeri M.Y.: Development of process control in sheet metal forming, Journal of Materials Processing Technology, 127 (2002), 3, 361-368.

[2] Bao J., Liu H., Xing Z.: Springback of hot stamping and die quenching with ultra-highstrength boron steel. Engineering Review, 33(2013), 3, 151-156.

[3] Liu N., Yang H., Li H.: Plastic wrinkling prediction in thin-walled part forming process: A review. Chinese Journal of Aeronautics, 29 (2016), 1, 1-14.

[4] Gao, T.J., Liu, Y., Wang, Z.J.: Research on process window diagrams of tube-compression by viscous pressure forming. Journal of Materials Engineering and Performance, 23 (2014), 6, 2020-2024.

[5] Wang, Z.J., Li, Y., Liu, J.G.: Evaluation of forming limit in viscous pressure forming of automotive aluminum alloy 6k21-T4 sheet. Trans. Nonferrous Met. Soc. China, 17 (2007), 6, 1169-1174.

[6] Foudeh, M.R., Daneshmand, S., Demirci, HI.: Investigating aluminum sheet wrinkling during the deep drawing process, Transactions of Famena, XXXVII (2013), 4, 43-54.

[7] Wang, C.T., Kinzel, G., Altan, T.: Wrinkling criterion for an anisotropic shell with compound curvatures in sheet forming. International Journal of Mechanical Sciences, 36 (1994), 10, 945-960.

[8] Wang, X., Cao, J.: An Analytical prediction of flange wrinkling in sheet metal forming. Journal of Manufacturing Processes, 2 (2000), 2, 100-107.

[9] Lin, Y., Yang, H., Li, H.: Influences of Forming Parameters on Wrinkling in NC Thinwalled Tube Bending. Acta Aeronautica et Astronautica Sinica, 24 (2003), 5, 456-461.

[10] Kim, J.B., Yang, D.Y.: Finite Element Analysis of the Wrinkling Initiation and Growth in Modified Yoshida Buckling Test. Metals and Materials International, 4(1998), 4,640-647.
[11] Cao, J., Wang, X., Mills, F.J.: Characterization of sheet buckling subjected to controlled boundary constraints. Journal of Manufacturing Science \& Engineering, 124 (2002), 3, 493-501.

[12] Cheng, H.S., Cao, J., Yao, H.: Wrinkling behavior of laminated steel sheets. Journal of Materials Processing Technology, 151(2004), $1,133-140$.

[13] Schleich, R., Albiez, C.: Investigation on simulation of buckling of aluminium sheet alloys, 7th European LS-DYNA Conference, 2009.

[14] Limam, A., Lee, L.H., Corona, E.: Inelastic wrinkling and collapse of tubes under combined bending and internal pressure. International Journal of Mechanical Sciences, 52 (2010), 5, 637-647.

[15] Grizelj, B., Cumin, J., Ergić, T.: Application of Hydroforming Process in Sheet Metal Formation. Strojarstvo Časopis Za Teoriju I Praksu U Strojarstvu, 51(2009), 4, 333-340.

[16] Wang, Z.J., Gao, T.J.: Inner and outer pressure forming of nickel based super-alloy thin-walled part with variable diameter sections. Trans. Nonferrous Met. Soc. China, 18 (2008), 2, 285290.

[17] Li, M., Brazill, R.L., Chu, E.W.: Initiation and growth of wrinkling due to nonuniform tension in sheet metal forming. Experimental Mechanics, 40 (2000), 2, 180-189.

[18] Hayashi, H., Hirata, M., Yoshida, K.: Buckling behavior of a square specimen in the diagonal tensile test and its application. Journal of the Japan Society for Technology of Plasticity. 24(1978), 275, 1298-1304

[19] Yoshida, K.: Purposes and Features of the Yoshida Wrinkling Test. Journal of the Japan Society for Technology of Plasticity, 272 (1983), 24, 901-908

[20] Wan, M., Yang, Y.Y., Ma, L.X.: Expression, Measurement and Calculation of the Sidewall Wrinkle for Axisymmetrical Curved Parts. Material Science \& Technology, 3(1995), 4, 100-103. 